\title{
Response of Four Market Classes of Dry Beans to Halosulfuron Applied Postemergence at Five Application Timings
}

\author{
Nader Soltani*, Christy Shropshire, Peter H. Sikkema \\ University of Guelph Ridgetown CampusRidgetown, Ontario, Canada \\ Email: soltanin@uoguelph.ca
}

Received 30 January 2015; accepted 23 February 2015; published 26 February 2015

Copyright (C) 2015 by authors and Scientific Research Publishing Inc.

This work is licensed under the Creative Commons Attribution International License (CC BY). http://creativecommons.org/licenses/by/4.0/

(c) (7) Open Access

\section{Abstract}

Six field trials were conducted over a three-year period $(2011,2012,2013)$ at the Huron Research Station, Exeter, Ontario and University of Guelph Ridgetown Campus, Ridgetown, Ontario to determine the effect of five postemergence (POST) application timings ( 1 - 2 trifoliate, 3 - 4 trifoliate, 5 6 trifoliate, $1^{\text {st }}$ flower and $1^{\text {st }}$ pod stage) of halosulfuron (35 and $70 \mathrm{~g}^{-a i} \cdot \mathrm{ha}^{-1}$ ) on the tolerance of adzuki, black, white and kidney beans. All treatments including the non-treated control were maintained weed free during the growing season. Halosulfuron applied POST in black, white and kidney bean caused as much as $8 \%, 8 \%, 7 \%, 4 \%$ and $2 \%$ injury 1 WAA and $4 \%, 4 \%, 2 \%, 2 \%$ and $1 \%$ injury 2 WAA at 1 - 2 trifoliate, 3 - 4 trifoliate, 5 - 6 trifoliate, $1^{\text {st }}$ flower and $1^{\text {st }}$ pod stage application timings, respectively. The injury observed was transient with $1 \%$ or less injury 4 WAA and there was no adverse effect on the shoot dry weight, plant height, seed moisture content and yield of black, white and kidney bean. Injury was substantially higher in adzuki bean at all application timings. Halosulfuron applied POST caused as much as $66 \%, 47 \%, 50 \%, 39 \%$ and $36 \%$ injury 1 WAA; $77 \%, 68 \%, 64 \%, 51 \%$ and $42 \% 2$ WAA; and $69 \%, 51 \%, 47 \%, 40 \%$ and $29 \% 4$ WAA at 1 - 2 trifoliate, 3 - 4 trifoliate, 5 - 6 trifoliate, $1^{\text {st }}$ flower and $1^{\text {st }}$ pod stage application timings, respectively. Halosulfuron POST decreased shoot dry weight as much as $69 \%, 57 \%, 43 \%, 41 \%$ and $34 \%$; plant height as much as $17 \%, 15 \%, 14 \%, 13 \%$ and $10 \%$; and seed yield as much as $47 \%, 46 \%, 45 \%$, $56 \%$ and $55 \%$ at 1 - 2 trifoliate, 3 - 4 trifoliate, 5 - 6 trifoliate, $1^{\text {st }}$ flower and $1^{\text {st }}$ pod stages, respectively. Based on these results, there is an adequate margin of crop safety for halosulfuron POST in black, white and kidney beans. However, there is not an adequate margin of crop safety for halosulfuron POST in adzuki bean at the application timings evaluated.

\section{Keywords}

Adzuki Bean, Black Bean, Kidney Bean, White Bean, Phaseolus vulgaris L.

\footnotetext{
${ }^{*}$ Corresponding author.
} 


\section{Introduction}

Edible dry beans (Phaseolus vulgaris L.) are a short season crop with short physical stature and therefore are very sensitive to weed interference [1]-[3]. Beans are more susceptible to yield losses due to weed interference than the other major field crops grown in Ontario. Bean seed yield has been reduced an average of 59\% in research studies conducted in Ontario. This is greater than the other major field crops: corn (52), soybean (38\%), spring cereals (12\%) and winter wheat (3\%). In addition, presence of weeds in beans at harvest can also cause seed staining and interfere with harvesting efficiency [4]-[6]. There are numerous broadleaf herbicides registered for use in soybean, but most of them cannot be used in edible beans because of crop injury. Consequently there is only one registered soil applied broadleaf herbicide-imazethapyr, and two postemergence (POST) broadleaf herbicides-bentazon and fomesafen. In contrast, Identity Preserved soybean producers have at least 12 broadleaf herbicides to choose from-acifluorfen, bentazon, chlorimuron, clomazone, cloransulam, flumetsulam, flumioxazin, fomesafen, imazethapyr, linuron, metribuzin and thifensulfuron [7]. Even with the wide array of herbicides registered for use in IP soybeans broadleaf weed control is still a challenge! This highlights the difficulty facing Ontario edible bean producers. Clearly, there is a lack of weed management tools for broadleaf weed control in edible beans. More research is needed to find new herbicide options that have an adequate margin of crop safety, provide consistent broad spectrum weed control, have low environmental impact and maximize bean yield and net returns.

Halosulfuron is a newly registered sulfonylurea herbicide in Ontario that inhibits the acetolactate synthase enzyme and blocks biosynthesis of key amino acids valine, leucine and isoleucine which are required for cell growth [8]. It is absorbed by roots, emerging shoots and foliage and is translocated in both xylem and phloem. Halosulfuron controls troublesome weeds including yellow nutsedge (Cyperus esculentus L.), velvetleaf (Abutilon theophrasti Medic.), redroot pigweed (Amaranthus retroflexus L.), common lambsquarters (Chenopodium album L.), ladysthumb (Polygonum persicaria L.), cocklebur (Xanthium strumarium L.) and wild mustard (Sinapis arvensis L.), including triazine resistant biotypes [7] [8]. Halosulfuron is active at low doses, has low mammalian toxicity, is relatively soil immobile and degrades rapidly, therefore has low potential to contaminate groundwater and the environment [8].

Halosulfuron will be available for the first time in Ontario in 2014. Halosulfuron will provide Ontario dry bean growers with a new, low-use-rate herbicide that provides full-season control of annual broadleaf weeds and specific troublesome weeds such as yellow nutsedge. There is little information available on the sensitivity of Phaseolus vulgaris (common bean) and Vigna angularis (adzuki bean) species to halosulfuron applied postemergence. Earlier studies have shown that halosulfuron applied POST at $2-3$ trifoliate can cause as much as 86\% injury in adzuki bean and as much as $13 \%$ injury in common bean [9]-[11]. There is little information with halosulfuron applied POST beyond the 3 trifoliate leaf stage in adzuki and common beans. Delaying application timing may reduce injury and provide an adequate crop safety for use of halosulfuron in dry bean.

The objective of this study was to evaluate the effect of halosulfuron applied postemergence at 35 and 70 $\mathrm{g} \cdot \mathrm{ai} \cdot \mathrm{ha}^{-1}$ at $1-2$ trifoliate, 3 - 4 trifoliate, $5-6$ trifoliate, $1^{\text {st }}$ flower and $1^{\text {st }}$ pod application timings in adzuki, black, white and kidney beans.

\section{Materials and Methods}

Six field trials were conducted over a three-year period (2011, 2012, and 2013) at the Huron Research Station,

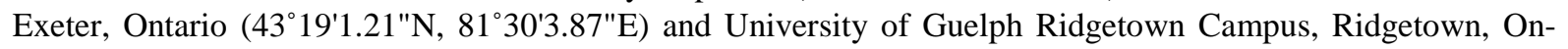
tario $\left(42^{\circ} 26^{\prime} 26^{\prime \prime} \mathrm{N}, 81^{\circ} 53^{\prime} 3^{\prime \prime} \mathrm{W}\right)$. The soil at Exeter was a Brookston clay loam (Orthic Humic Gleysol, mixed, mesic, and poorly drained) with $32 \%$ sand, $42 \%$ silt, $26 \%$ clay, $3.7 \%$ organic matter and $\mathrm{pH} 7.8$ in 2011; $41 \%$ sand, 35\% silt, 24\% clay, 3.2\% organic matter and pH 7.9 in 2012; and $29 \%$ sand, $44 \%$ silt, $27 \%$ clay, 3.6\% organic matter and $\mathrm{pH} 7.7$ in 2013. The soil at the Ridgetown location was a Watford/Brady sandy loam composed of $48 \%$ sand, $28 \%$ silt, $24 \%$ clay, and $6.7 \%$ organic matter with a pH of 6.6 in $2011 ; 49 \%$ sand, $31 \%$ silt, $20 \%$ clay, $6.0 \%$ organic matter and pH 6.5 in 2012; and 52\% sand, 28\% silt, $20 \%$ clay, 5.9\% organic matter and pH 6.4 in 2013. Seedbed preparation at all sites consisted of fall moldboard plowing followed by three passes with a field cultivator with rolling basket harrows in the spring.

The experiments were established as a two-way factorial in a completely randomized block design with four replications. Factor one was market class of dry bean (black, "Black Velvet”; white, "T9905”; adzuki, "Erimo”; and kidney, "Red Hawk") and Factor 2 was herbicide treatment (Halosulfuron applied POST at $35 \mathrm{~g} \cdot \mathrm{ai}^{\mathrm{i}} \mathrm{ha}^{-1}$ or 
sprayed twice to simulate a spray overlap at each application timing: 1 - 2 trifoliate, 3 - 4 trifoliate, 5 - 6 trifoliate, 1 st flower, 1st pod stage plus a non-treated control). Halosulfuron treatments included a non-ionic surfactant at $0.25 \% \mathrm{v} / \mathrm{v}$. Plots were $6 \mathrm{~m}$ wide (8 rows spaced $0.75 \mathrm{~m}$ apart) and $10 \mathrm{~m}$ long at Exeter and $8 \mathrm{~m}$ long at Ridgetown. Within each plot there were two rows of black, "Black Velvet”; white, "T9905”; adzuki, "Erimo"; and kidney, "Red Hawk" beans. Beans were planted $3 \mathrm{~cm}$ deep at the rate of 175,000 seed·ha ${ }^{-1}$ for kidney bean and 230,000 seed $\cdot \mathrm{ha}^{-1}$ for black, white and adzuki bean in late May to early June of each year.

Herbicide applications at each timing ( 1 - 2 trifoliate, 3 - 4 trifoliate, 5 - 6 trifoliate, $1^{\text {st }}$ flower, $1^{\text {st }}$ pod) were made with a $\mathrm{CO}_{2}$-pressurized backpack sprayer calibrated to deliver $200 \mathrm{~L} \cdot \mathrm{ha}^{-1}$ of spray solution at a pressure of 200/241 kPa using low drift nozzles (ULD120-02, Spraying Systems Co., P.O. Box 7900. Wheaton, IL 60188). The boom was $2.5 \mathrm{~m}$ wide with six nozzles spaced $0.5 \mathrm{~m}$ apart. Plots were maintained weed free by cultivation and hand hoeing as required to eliminate the confounding effect of weed interference.

Crop injury was evaluated visually 1, 2 and 4 weeks after each treatment application (WAA) using a scale of $0 \%$ to $100 \%$ where a rating of 0 was defined as no visible plant injury and a rating of 100 was defined as plant death. At 2 WAA, a $1 \mathrm{~m}$ section of row for each cultivar was hand harvested at the ground level, oven dried at $60^{\circ} \mathrm{C}$ to constant moisture and the dry weight was recorded. Ten plants per plot were randomly selected and the height from the soil surface to the highest growing point was measured 5 WAA. Yield and seed moisture content were measured at crop maturity by harvesting the remaining $9 \mathrm{~m}$ from each plot at Exeter and $7 \mathrm{~m}$ from each plot at Ridgetown with a plot combine. Crops were considered physically mature when $90 \%$ of pods in the untreated plots of each cultivar had turned from green to a golden colour. All yields were adjusted to $18 \%$ moisture.

Data were analyzed as a 2-way factorial using PROC MIXED in SAS 9.2. Fixed effects included the two treatment factors, dry bean market class and halosulfuron treatment, as well as their interaction; random effects included year-location combinations (environment), interactions between environment and the fixed effects, and replicate nested within environment. Significance of fixed effects was tested using F-tests and random effects were tested using a Z-test of the variance estimate. The UNIVARIATE procedure was used to test data for normality and homogeneity of variance. To satisfy the assumptions of the variance analyses, injury 1, 2 and 4 WAA was arcsine square root transformed, percent dry weight were square root transformed and seed moisture content at harvest was log-transformed. For all injury ratings, the untreated check (assigned a value of zero) was excluded from the analysis. However, all values were compared independently to zero to evaluate treatment differences with the untreated check. Plant stand, shoot dry weight, height and yield were converted to a percent of the untreated check for analysis. Treatment comparisons were made using Fisher's Protected LSD at a level of P < 0.05 and any data compared on the transformed scale were converted back to the original scale for presentation of results. When the interactions between location, year and fixed effects were not significant and the data were pooled by location and year.

\section{Results and Discussion}

Analysis of variance indicated that for main effects, herbicide treatment was significant for injury 1, 2 and 4 WAA, shoot dry weight, seed moisture content and yield (Table 1). Market class was significant for injury 1, 2 and 4 WAA, shoot dry weight, height, and yield (Table 1). For interactions, cultivar by treatment was significant for injury 1, 2 and 4 WAA, shoot dry weight, height, seed moisture content and yield (Table 1). Injury symptoms with halosulfuron included chlorosis, necrosis, stunting and death of the growing point of dry bean (Table 1).

\subsection{Crop Injury}

Halosulfuron applied POST in black, white and kidney bean caused as much as 8\%, 8\%, 7\%, 4\% and 2\% injury 1 WAA and 4\%, 4\%, 2\%, 2\% and 1\% injury 2 WAA at $1-2$ trifoliate, $3-4$ trifoliate, $5-6$ trifoliate, $1^{\text {st }}$ flower and $1^{\text {st }}$ pod stage application timings, respectively (Table 2). However by 4 WAA, there was no injury in black and white and up to 1\% injury in kidney bean with halosulfuron applied POST at all application timings (Table 2).

Injury was significantly higher in adzuki bean compared to black, white and kidney bean at all application timings. At 1 WAA, halosulfuron applied POST caused as much as 66\%, 47\%, 50\%, 39\% and 36\% injury at 1 2 trifoliate, 3 - 4 trifoliate, 5 - 6 trifoliate, $1^{\text {st }}$ flower and $1^{\text {st }}$ pod application timings in adzuki bean, respectively (Table 2). Injury was persistent and did not decrease over time. Adzuki bean injury was as much as $77 \%, 68 \%$, 
Table 1. Main effects and interaction for percent visible injury, plant stand, height, shoot dry weight, seed moisture content and yield of dry bean treated with halosulfuron at five different timings. Plant stand, height, shoot dry weight and yield are a percent of the untreated check. Means followed by the same letter within a column are not significantly different according to Fisher's Protected LSD at $\mathrm{P}<0.05$. Means for a main effect were separated only if there was no significant interaction involving that main effect ${ }^{\mathrm{a}}$.

\begin{tabular}{|c|c|c|c|c|c|c|c|c|c|c|}
\hline \multicolumn{11}{|c|}{ Dry bean injury } \\
\hline Main effects ${ }^{b}$ & & & 1 WAA & 2 WAA & 4 WAA & $\begin{array}{l}\text { Plant } \\
\text { stand }\end{array}$ & $\begin{array}{c}\text { Dry } \\
\text { weight }\end{array}$ & Height & Moisture & Yield \\
\hline \multicolumn{11}{|c|}{$\%$} \\
\hline Dry bean market class & & & $* *$ & $* *$ & ** & NS & ** & $* *$ & NS & $* *$ \\
\hline Black & & & 2 & 1 & 0 & 99 & 100 & 102 & 18.1 & 97 \\
\hline White & & & 2 & 1 & 0 & 102 & 105 & 100 & 19.1 & 110 \\
\hline Adzuki & & & 45 & 57 & 40 & 100 & 60 & 89 & 18.1 & 61 \\
\hline Kidney & & & 4 & 2 & 0 & 108 & 109 & 104 & 19.4 & 109 \\
\hline Herbicide treatment ${ }^{\mathrm{c}}$ & $\begin{array}{c}\text { Rate } \\
\left(\mathrm{g} \cdot \mathrm{ai} \cdot \mathrm{ha}^{-1}\right)\end{array}$ & Timing & $* *$ & ** & ** & NS & ** & NS & $* *$ & $*$ \\
\hline Untreated check & & & 0 & 0 & 0 & 100 & 100 & 100 & 17.5 & 100 \\
\hline Halosulfuron & 35 & $1-2$ tri & 12 & 11 & 6 & 97 & 87 & 98 & 18.1 & 96 \\
\hline Halosulfuron & 70 & $1-2$ tri & 18 & 16 & 9 & 97 & 81 & 97 & 18.9 & 93 \\
\hline Halosulfuron & 35 & $3-4$ tri & 9 & 10 & 4 & 106 & 94 & 99 & 18.0 & 98 \\
\hline Halosulfuron & 70 & $3-4$ tri & 13 & 13 & 6 & 107 & 86 & 97 & 18.3 & 94 \\
\hline Halosulfuron & 35 & $5-6$ tri & 9 & 8 & 3 & 103 & 93 & 100 & 18.2 & 96 \\
\hline Halosulfuron & 70 & $5-6$ tri & 11 & 9 & 5 & 103 & 92 & 98 & 18.6 & 93 \\
\hline Halosulfuron & 35 & $1^{\text {st }}$ flower & 5 & 5 & 3 & 104 & 100 & 100 & 18.9 & 94 \\
\hline Halosulfuron & 70 & $1^{\text {st }}$ flower & 7 & 7 & 4 & 106 & 94 & 100 & 19.5 & 91 \\
\hline Halosulfuron & 35 & $1^{\text {st }}$ pod & 4 & 4 & 2 & 101 & 97 & 100 & 19.6 & 91 \\
\hline Halosulfuron & 70 & $1^{\text {st }}$ pod & 6 & 5 & 3 & 102 & 94 & 99 & 19.8 & 88 \\
\hline \multicolumn{11}{|l|}{ Interaction } \\
\hline $\mathrm{V} \times \mathrm{H}$ & & & ** & ** & $* *$ & NS & $* *$ & $* *$ & $* *$ & $* *$ \\
\hline
\end{tabular}

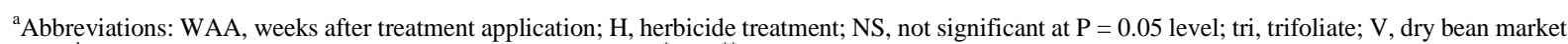
class. ${ }^{\mathrm{b}}$ Significance at $\mathrm{P}<0.05$ and $\mathrm{P}<0.01$ levels denoted by ${ }^{*}$ and ${ }^{* *}$, respectively. ${ }^{\mathrm{C}}$ Non-ionic surfactant included at $0.25 \%$ and $0.5 \% \mathrm{v} / \mathrm{v}$ with the 35 and $70 \mathrm{~g} \cdot \mathrm{ai} \cdot \mathrm{ha}^{-1}$ of halosulfuron, respectively.

64\%, 51\% and 42\% 2 WAA and 69\%, 51\%, 47\%, 40\% and 29\% 4 WAA at 1 - 2 trifoliate, 3 - 4 trifoliate, 5 - 6 trifoliate, $1^{\text {st }}$ flower and $1^{\text {st }}$ pod application timings, respectively (Table 2).

In other studies, halosulfuron applied POST at 2 - 3 trifoliate caused as much as $73 \%, 7 \%, 13 \%, 12 \%, 12 \%$, $11 \%, 11 \%$ and $9 \%$ injury in adzuki, black, cranberry, kidney, otebo, pinto, small red Mexican and white beans, respectively [9] [10]. Stewart et al. [10] found as much as $67 \%$ and $86 \%$ injury when halosulfuron was applied POST at $35 \mathrm{~g} \cdot$ ai $\cdot \mathrm{ha}^{-1}$ and $70 \mathrm{~g} \cdot \mathrm{ai} \cdot \mathrm{ha}^{-1}$ in adzuki bean, respectively. Wall [12] also reported as much as $50 \%$ injury in white bean with halosulfuron applied POST. Silvey et al. [13] reported 5\% injury from halosulfuron POST in snap bean. Other sulfonylurea herbicides such as thifensulfuron have been shown to cause up to $67 \%$ injury in some market classes of beans [10].

\subsection{Shoot Dry Weight}

Halosulfuron applied POST at 35 and $70 \mathrm{~g} \cdot \mathrm{ai} \cdot \mathrm{ha}^{-1}$ had no adverse effect on the shoot dry weight of black, white and kidney bean at all application timings (Table 3). However, it decreased adzuki bean shoot dry weight as much as $69 \%, 57 \%, 43 \%, 41 \%$ and $34 \%$ at $1-2$ trifoliate, $3-4$ trifoliate, $5-6$ trifoliate, $1^{\text {st }}$ flower and $1^{\text {st }}$ pod 
Table 2. Percent visible injury 1, 2 and 4 WAA for four dry bean market classes treated with halosulfuron at five different timings. Means followed by the same letter within a column (a-g) or row (Y-Z) are not significantly different according to Fisher's Protected LSD at $\mathrm{P}<0.05^{\mathrm{a}}$.

\begin{tabular}{|c|c|c|c|c|c|c|c|c|c|c|c|c|c|c|}
\hline $\begin{array}{l}\text { Herbicide } \\
\text { treatment }^{\mathrm{b}}\end{array}$ & $\begin{array}{c}\text { Rate } \\
\left(\mathrm{g} \cdot \mathrm{ai} \cdot \mathrm{ha}^{-1}\right)\end{array}$ & Timing & & Black & & & White & & & Adzuki & & & Kidney & \\
\hline Injury 1 WAA & & & & & & & & $\%$ & & & & & & \\
\hline Untreated check & & & 0 & $\mathrm{a}$ & & 0 & $\mathrm{a}$ & & 0 & $\mathrm{a}$ & & 0 & $\mathrm{a}$ & \\
\hline Halosulfuron & 35 & $1-2$ tri & 3 & $\mathrm{~cd}$ & $\mathrm{Z}$ & 4 & cde & $\mathrm{Z}$ & 60 & fg & $\mathrm{Y}$ & 3 & bc & $\mathrm{Z}$ \\
\hline Halosulfuron & 70 & $1-2$ tri & 6 & $\mathrm{~d}$ & $\mathrm{Z}$ & 8 & e & $\mathrm{Z}$ & 66 & g & $\mathrm{Y}$ & 6 & $\mathrm{~cd}$ & $\mathrm{Z}$ \\
\hline Halosulfuron & 35 & $3-4$ tri & 2 & bc & $\mathrm{Z}$ & 3 & bcd & $\mathrm{Z}$ & 40 & bcd & $\mathrm{Y}$ & 4 & bcd & $\mathrm{Z}$ \\
\hline Halosulfuron & 70 & $3-4$ tri & 4 & $\mathrm{~cd}$ & $\mathrm{Z}$ & 6 & de & $\mathrm{Z}$ & 47 & de & $\mathrm{Y}$ & 8 & d & $\mathrm{Z}$ \\
\hline Halosulfuron & 35 & $5-6$ tri & 1 & abc & $\mathrm{Z}$ & 2 & bc & $\mathrm{Z}$ & 46 & cde & $\mathrm{Y}$ & 5 & $\mathrm{~cd}$ & $\mathrm{Z}$ \\
\hline Halosulfuron & 70 & $5-6$ tri & 2 & bc & $\mathrm{Z}$ & 3 & bcd & $\mathrm{Z}$ & 50 & ef & $\mathrm{Y}$ & 7 & $\mathrm{~cd}$ & $\mathrm{Z}$ \\
\hline Halosulfuron & 35 & $1^{\text {st }}$ flower & 0 & $\mathrm{ab}$ & $\mathrm{Z}$ & 0 & $\mathrm{a}$ & $\mathrm{Z}$ & 34 & $\mathrm{~b}$ & $\mathrm{Y}$ & 3 & bc & $\mathrm{Z}$ \\
\hline Halosulfuron & 70 & $1^{\text {st }}$ flower & 1 & $\mathrm{abc}$ & $\mathrm{Z}$ & 2 & bc & $\mathrm{Z}$ & 39 & bcd & $\mathrm{Y}$ & 4 & bcd & $\mathrm{Z}$ \\
\hline Halosulfuron & 35 & $1^{\text {st }}$ pod & 0 & $\mathrm{ab}$ & $\mathrm{Z}$ & 0 & $\mathrm{a}$ & $\mathrm{Z}$ & 31 & $\mathrm{~b}$ & $\mathrm{Y}$ & 1 & b & $\mathrm{Z}$ \\
\hline Halosulfuron & 70 & $1^{\text {st }}$ pod & 0 & $\mathrm{ab}$ & $\mathrm{Z}$ & 1 & $\mathrm{ab}$ & $\mathrm{Z}$ & 36 & bc & $\mathrm{Y}$ & 2 & bc & $\mathrm{Z}$ \\
\hline Injury 2 WAA & & & & & & & & $\%$ & & & & & & \\
\hline Untreated check & & & 0 & $\mathrm{a}$ & & 0 & $\mathrm{a}$ & & 0 & $\mathrm{a}$ & & 0 & $\mathrm{a}$ & \\
\hline Halosulfuron & 35 & $1-2$ tri & 1 & $\mathrm{ab}$ & $\mathrm{Z}$ & 2 & bc & $\mathrm{Z}$ & 71 & fg & $\mathrm{Y}$ & 1 & b & $\mathrm{Z}$ \\
\hline Halosulfuron & 70 & $1-2$ tri & 3 & $\mathrm{~b}$ & $\mathrm{Z}$ & 4 & c & $\mathrm{Z}$ & 77 & g & $\mathrm{Y}$ & 3 & bc & $\mathrm{Z}$ \\
\hline Halosulfuron & 35 & $3-4$ tri & 1 & $\mathrm{ab}$ & $\mathrm{Z}$ & 1 & $a b$ & $\mathrm{Z}$ & 59 & de & $\mathrm{Y}$ & 3 & bc & $\mathrm{Z}$ \\
\hline Halosulfuron & 70 & $3-4$ tri & 2 & $\mathrm{~b}$ & $\mathrm{Z}$ & 3 & bc & $\mathrm{Z}$ & 68 & $\mathrm{f}$ & $\mathrm{Y}$ & 4 & c & $\mathrm{Z}$ \\
\hline Halosulfuron & 35 & $5-6$ tri & 0 & $\mathrm{a}$ & $\mathrm{Z}$ & 1 & $\mathrm{ab}$ & $\mathrm{Z}$ & 56 & de & $\mathrm{Y}$ & 2 & bc & $\mathrm{Z}$ \\
\hline Halosulfuron & 70 & $5-6$ tri & 1 & $\mathrm{ab}$ & $\mathrm{Z}$ & 1 & $\mathrm{ab}$ & $\mathrm{Z}$ & 64 & ef & $\mathrm{Y}$ & 2 & bc & $\mathrm{Z}$ \\
\hline Halosulfuron & 35 & $1^{\text {st }}$ flower & 0 & $\mathrm{a}$ & $\mathrm{Z}$ & 0 & a & $\mathrm{Z}$ & 42 & bc & $\mathrm{Y}$ & 1 & $\mathrm{~b}$ & $\mathrm{Z}$ \\
\hline Halosulfuron & 70 & $1^{\text {st }}$ flower & 0 & $\mathrm{a}$ & $\mathrm{Z}$ & 1 & $a b$ & $\mathrm{Z}$ & 51 & $\mathrm{~cd}$ & $\mathrm{Y}$ & 2 & bc & $\mathrm{Z}$ \\
\hline Halosulfuron & 35 & $1^{\text {st }}$ pod & 0 & a & $\mathrm{Z}$ & 0 & a & $\mathrm{Z}$ & 34 & $\mathrm{~b}$ & $\mathrm{Y}$ & 1 & $\mathrm{~b}$ & $\mathrm{Z}$ \\
\hline Halosulfuron & 70 & $1^{\text {st }}$ pod & 0 & $\mathrm{a}$ & $\mathrm{Z}$ & 1 & $a b$ & $\mathrm{Z}$ & 42 & bc & $\mathrm{Y}$ & 1 & b & $\mathrm{Z}$ \\
\hline Injury 4 WAA & & & & & & & & $\%$ & & & & & & \\
\hline Untreated check & & & 0 & $\mathrm{a}$ & & 0 & $\mathrm{a}$ & & 0 & $\mathrm{a}$ & & 0 & a & \\
\hline Halosulfuron & 35 & $1-2$ tri & 0 & $\mathrm{a}$ & $\mathrm{Z}$ & 0 & a & $\mathrm{Z}$ & 47 & ef & $\mathrm{Y}$ & 1 & $\mathrm{~b}$ & $\mathrm{Z}$ \\
\hline Halosulfuron & 70 & $1-2$ tri & 0 & $\mathrm{a}$ & $\mathrm{Z}$ & 0 & a & $\mathrm{Z}$ & 69 & g & $\mathrm{Y}$ & 1 & $\mathrm{~b}$ & $\mathrm{Z}$ \\
\hline Halosulfuron & 35 & $3-4$ tri & 0 & $\mathrm{a}$ & $\mathrm{Z}$ & 0 & a & $\mathrm{Z}$ & 35 & $\mathrm{~cd}$ & $\mathrm{Y}$ & 0 & $\mathrm{a}$ & $\mathrm{Z}$ \\
\hline Halosulfuron & 70 & $3-4$ tri & 0 & $\mathrm{a}$ & $\mathrm{Z}$ & 0 & $\mathrm{a}$ & $\mathrm{Z}$ & 51 & $\mathrm{f}$ & $\mathrm{Y}$ & 1 & $\mathrm{~b}$ & $\mathrm{Z}$ \\
\hline Halosulfuron & 35 & $5-6$ tri & 0 & $\mathrm{a}$ & $\mathrm{Z}$ & 0 & $\mathrm{a}$ & $\mathrm{Z}$ & 36 & $\mathrm{~cd}$ & $\mathrm{Y}$ & 0 & $\mathrm{a}$ & $\mathrm{Z}$ \\
\hline Halosulfuron & 70 & $5-6$ tri & 0 & $\mathrm{a}$ & $\mathrm{Z}$ & 0 & $\mathrm{a}$ & $\mathrm{Z}$ & 47 & ef & $\mathrm{Y}$ & 0 & $\mathrm{a}$ & $\mathrm{Z}$ \\
\hline Halosulfuron & 35 & $1^{\text {st }}$ flower & 0 & a & $\mathrm{Z}$ & 0 & a & $\mathrm{Z}$ & 29 & bc & $\mathrm{Y}$ & 0 & a & $\mathrm{Z}$ \\
\hline Halosulfuron & 70 & $1^{\text {st }}$ flower & 0 & $\mathrm{a}$ & $\mathrm{Z}$ & 0 & $\mathrm{a}$ & $\mathrm{Z}$ & 40 & de & $\mathrm{Y}$ & 0 & $\mathrm{a}$ & $\mathrm{Z}$ \\
\hline Halosulfuron & 35 & $1^{\text {st }}$ pod & 0 & $\mathrm{a}$ & $\mathrm{Z}$ & 0 & a & $\mathrm{Z}$ & 23 & $\mathrm{~b}$ & $\mathrm{Y}$ & 0 & a & $\mathrm{Z}$ \\
\hline Halosulfuron & 70 & $1^{\text {st }}$ pod & 0 & $\mathrm{a}$ & $\mathrm{Z}$ & 0 & a & $\mathrm{Z}$ & 29 & bc & $\mathrm{Y}$ & 0 & a & $\mathrm{Z}$ \\
\hline
\end{tabular}

${ }^{\mathrm{a}}$ Abbreviations: WAA, weeks after treatment application; tri, trifoliate. ${ }^{\mathrm{b}}$ Non-ionic surfactant included at $0.25 \%$ and $0.5 \% \mathrm{v} / \mathrm{v}$ with the 35 and 70 $\mathrm{g} \cdot \mathrm{g} \cdot \mathrm{ai} \cdot \mathrm{ha}^{-1}$ of halosulfuron, respectively. 
Table 3. Shoot dry weight (2 WAA) and height (5 WAA), both as a percent of the untreated check, for four dry bean market classes treated with halosulfuron at five different timings. Means followed by the same letter within a column (a-g) or row (X-Z) are not significantly different according to Fisher's Protected LSD at $\mathrm{P}<0.05^{\mathrm{a}}$.

\begin{tabular}{|c|c|c|c|c|c|c|c|c|c|c|c|c|c|c|}
\hline \multirow{2}{*}{$\begin{array}{c}\begin{array}{c}\text { Herbicide } \\
\text { treatment }^{\mathrm{b}}\end{array} \\
\text { Shoot dry weight }\end{array}$} & $\begin{array}{c}\text { Rate } \\
\left(\mathrm{g} \cdot \mathrm{ai} \cdot \mathrm{ha}^{-1}\right)\end{array}$ & Timing & \multicolumn{3}{|c|}{ Black } & \multicolumn{3}{|c|}{ White } & \multicolumn{3}{|c|}{ Adzuki } & \multicolumn{3}{|c|}{ Kidney } \\
\hline & & & & & & & & $\%$ & & & & & & \\
\hline Untreated check & & & 100 & a & & 100 & a & & 100 & a & & 100 & $\mathrm{~b}$ & \\
\hline Halosulfuron & 35 & $1-2$ tri & 101 & a & $\mathrm{Z}$ & 101 & a & $\mathrm{Z}$ & 50 & ef & $\mathrm{Z}$ & 104 & $\mathrm{~b}$ & $\mathrm{Z}$ \\
\hline Halosulfuron & 70 & $1-2$ tri & 96 & a & $\mathrm{Z}$ & 105 & $\mathrm{a}$ & $\mathrm{Z}$ & 31 & g & $\mathrm{Z}$ & 106 & $\mathrm{ab}$ & $\mathrm{Z}$ \\
\hline Halosulfuron & 35 & $3-4$ tri & 100 & a & $\mathrm{Z}$ & 111 & a & $\mathrm{Z}$ & 61 & cde & $\mathrm{Z}$ & 111 & $\mathrm{ab}$ & $\mathrm{Z}$ \\
\hline Halosulfuron & 70 & $3-4$ tri & 98 & a & $\mathrm{Z}$ & 105 & $\mathrm{a}$ & $\mathrm{Z}$ & 43 & $\mathrm{f}$ & $\mathrm{Z}$ & 108 & $\mathrm{ab}$ & $\mathrm{Z}$ \\
\hline Halosulfuron & 35 & $5-6$ tri & 103 & a & $\mathrm{Z}$ & 102 & a & $\mathrm{Z}$ & 64 & bcd & $\mathrm{Z}$ & 108 & $a b$ & $\mathrm{Z}$ \\
\hline Halosulfuron & 70 & $5-6$ tri & 99 & $\mathrm{a}$ & $\mathrm{Z}$ & 105 & a & $\mathrm{Z}$ & 57 & de & $\mathrm{Z}$ & 114 & $\mathrm{ab}$ & $\mathrm{Z}$ \\
\hline Halosulfuron & 35 & $1^{\text {st }}$ flower & 107 & a & $\mathrm{Z}$ & 110 & a & $\mathrm{Z}$ & 70 & bc & $\mathrm{Z}$ & 116 & $\mathrm{ab}$ & $\mathrm{Z}$ \\
\hline Halosulfuron & 70 & $1^{\text {st }}$ flower & 98 & a & $\mathrm{Y}$ & 102 & $\mathrm{a}$ & $\mathrm{Y}$ & 59 & cde & $\mathrm{X}$ & 121 & $\mathrm{a}$ & $\mathrm{Z}$ \\
\hline Halosulfuron & 35 & $1^{\text {st }}$ pod & 96 & a & $\mathrm{Z}$ & 108 & a & $\mathrm{Z}$ & 77 & $\mathrm{~b}$ & $\mathrm{Z}$ & 108 & $a b$ & $\mathrm{Z}$ \\
\hline Halosulfuron & 70 & $1^{\text {st }}$ pod & 105 & a & $\mathrm{Z}$ & 104 & $\mathrm{a}$ & $\mathrm{Z}$ & 66 & bcd & $\mathrm{Z}$ & 106 & $\mathrm{ab}$ & $\mathrm{Z}$ \\
\hline Height & & & & & & & & $\%$ & & & & & & \\
\hline Untreated check & & & 100 & a & $\mathrm{Z}$ & 100 & a & $\mathrm{Z}$ & 100 & $\mathrm{a}$ & $\mathrm{Z}$ & 100 & c & $\mathrm{Z}$ \\
\hline Halosulfuron & 35 & $1-2$ tri & 101 & a & $\mathrm{Z}$ & 99 & a & $\mathrm{Z}$ & 88 & cde & $\mathrm{Z}$ & 102 & abc & $\mathrm{Z}$ \\
\hline Halosulfuron & 70 & $1-2$ tri & 101 & a & $\mathrm{Z}$ & 100 & a & $\mathrm{Z}$ & 83 & e & $\mathrm{Z}$ & 104 & $\mathrm{abc}$ & $\mathrm{Z}$ \\
\hline Halosulfuron & 35 & $3-4$ tri & 100 & a & $\mathrm{Z}$ & 99 & a & $\mathrm{Z}$ & 90 & bcd & $\mathrm{Z}$ & 105 & $a b$ & $\mathrm{Z}$ \\
\hline Halosulfuron & 70 & $3-4$ tri & 103 & a & $\mathrm{Z}$ & 101 & a & $\mathrm{Z}$ & 85 & e & $\mathrm{Z}$ & 101 & bc & $\mathrm{Z}$ \\
\hline Halosulfuron & 35 & $5-6$ tri & 103 & a & $\mathrm{Z}$ & 101 & a & $\mathrm{Z}$ & 91 & bc & $\mathrm{Z}$ & 104 & abc & $\mathrm{Z}$ \\
\hline Halosulfuron & 70 & $5-6$ tri & 102 & a & $\mathrm{Z}$ & 100 & a & $\mathrm{Z}$ & 86 & de & $\mathrm{Z}$ & 105 & $a b$ & $\mathrm{Z}$ \\
\hline Halosulfuron & 35 & $1^{\text {st }}$ flower & 103 & $\mathrm{a}$ & $\mathrm{Z}$ & 102 & $\mathrm{a}$ & $\mathrm{Z}$ & 91 & bc & $\mathrm{Z}$ & 105 & $\mathrm{ab}$ & $\mathrm{Z}$ \\
\hline Halosulfuron & 70 & $1^{\text {st }}$ flower & 103 & a & $\mathrm{Z}$ & 101 & a & $\mathrm{Z}$ & 87 & cde & $\mathrm{Z}$ & 107 & a & $\mathrm{Z}$ \\
\hline Halosulfuron & 35 & $1^{\text {st }}$ pod & 103 & a & $\mathrm{Z}$ & 100 & a & $\mathrm{YZ}$ & 92 & $\mathrm{~b}$ & $\mathrm{Y}$ & 103 & abc & $\mathrm{Z}$ \\
\hline Halosulfuron & 70 & $1^{\text {st }}$ pod & 101 & a & $\mathrm{Z}$ & 99 & a & $\mathrm{Z}$ & 90 & bcd & $\mathrm{Z}$ & 105 & $\mathrm{ab}$ & $\mathrm{Z}$ \\
\hline
\end{tabular}

${ }^{\mathrm{a}}$ Abbreviations: WAA, weeks after treatment application; tri, trifoliate. ${ }^{\mathrm{b}}$ Non-ionic surfactant included at $0.25 \%$ and $0.5 \% \mathrm{v} / \mathrm{v}$ with the 35 and 70 $\mathrm{g} \cdot \mathrm{ai} \cdot \mathrm{ha}^{-1}$ of halosulfuron, respectively.

application timings, respectively (Table 3). In other studies, halosulfuron applied POST at 2 - 3 trifoliate reduced shoot dry weight of otebo bean 12\%, small red Mexican bean $12 \%$ and adzuki bean $68 \%$ but had no effects on shoot dry weight of black, cranberry, kidney, pinto and white beans at 35 and $70 \mathrm{~g} \cdot \mathrm{ai}^{\mathrm{h}} \mathrm{ha}^{-1}$ [9]. In another study, significant shoot dry weight reduction was seen with halosulfuron and thifensulfuron applied POST in adzuki bean [10]. Other sulfonylurea herbicides such as thifensulfuron and chorimuron applied POST have been shown to reduce shoot dry weight $27 \%$ - $64 \%$ in dry bean [14].

\subsection{Plant Height}

Height of beans is critical as beans are commonly direct harvested by combines and shorter plants tend to have greater shatter loss at the cutter bar of the combine resulting in reduced harvested seed yield.

Halosulfuron applied POST at 35 and $70 \mathrm{~g} \cdot \mathrm{ai} \cdot \mathrm{ha}^{-1}$ had no adverse effect on the height of black, white and kidney bean at all application timings (Table 3). However, it decreased adzuki bean height as much as $17 \%$, $15 \%, 14 \%, 13 \%$ and $10 \%$ at 1 - 2 trifoliate, 3 - 4 trifoliate, 5 - 6 trifoliate, $1^{\text {st }}$ flower and $1^{\text {st }}$ pod application tim- 
ings, respectively (Table 3). In other studies, halosulfuron applied POST at 2 - 3 trifoliate reduced adzuki bean height as much as $60 \%$ and $70 \%$ at 35 and $70 \mathrm{~g} \cdot \mathrm{ai}^{-h^{-1}}{ }^{-1}$, respectively but had no effect on the height of black, cranberry, kidney, otebo, pinto, small red Mexican and white beans [9]. This is in contrast to previous studies that have shown significant plant height reduction from sulfonylurea herbicides in dry bean. Thifensulfuron and halosulfuron caused significant reduction in height of adzuki bean [10]. Thifensulfuron applied POST decreased plant height $15 \%$ to $57 \%$ in dry bean [14]. Chlorimuron applied POST also decreased plant height as much as 36\% in white bean [14].

\subsection{Seed Moisture Content}

Quality of dry bean seeds can be affected by seed moisture content at harvest time as seeds coats can split when seed moisture is less than $18 \%$ and seeds can have increased respiration and be prone to spoilage at greater than $18 \%$ seed moisture content.

Halosulfuron applied POST at 35 and $70 \mathrm{~g} \cdot \mathrm{ai} \cdot \mathrm{ha}^{-1}$ had no effect on the seed moisture content of black, white and kidney bean at all application timings (Table 4). However, it increased adzuki bean seed moisture content as

Table 4. Seed moisture content at harvest, and yield as a percent of the untreated check for four dry bean market classes treated with halosulfuron at five different timings. Means followed by the same letter within a column (a-g) or row (X-Z) are not significantly different according to Fisher's Protected LSD at $\mathrm{P}<0.05^{\mathrm{a}}$.

\begin{tabular}{|c|c|c|c|c|c|c|c|c|c|c|c|c|c|c|}
\hline $\begin{array}{l}\text { Herbicide } \\
\text { treatment }^{\mathrm{b}}\end{array}$ & $\begin{array}{c}\text { Rate } \\
\left(\text { g ai ha }^{-1} \text { ) }\right.\end{array}$ & Timing & & Black & & & Thite & & & Adzuki & & & dney & \\
\hline Moisture & & & & & & & & $\%$ & & & & & & \\
\hline Untreated check & & & 18.2 & $\mathrm{a}$ & $\mathrm{Y}$ & 19.2 & $\mathrm{a}$ & $\mathrm{Y}$ & 14.0 & $\mathrm{a}$ & $\mathrm{Z}$ & 19.1 & $\mathrm{a}$ & $\mathrm{Y}$ \\
\hline Halosulfuron & 35 & $1-2$ tri & 18.1 & $\mathrm{a}$ & $\mathrm{YZ}$ & 19.0 & $\mathrm{a}$ & $\mathrm{Y}$ & 16.3 & $\mathrm{~b}$ & $\mathrm{Z}$ & 19.3 & $\mathrm{a}$ & $\mathrm{Y}$ \\
\hline Halosulfuron & 70 & $1-2$ tri & 18.2 & $\mathrm{a}$ & $\mathrm{Z}$ & 19.5 & $\mathrm{a}$ & $\mathrm{Z}$ & 18.4 & de & $\mathrm{Z}$ & 19.5 & $\mathrm{a}$ & $\mathrm{Z}$ \\
\hline Halosulfuron & 35 & $3-4$ tri & 17.7 & $\mathrm{a}$ & $\mathrm{YZ}$ & 19.0 & $\mathrm{a}$ & $\mathrm{Y}$ & 16.2 & $\mathrm{~b}$ & $\mathrm{Z}$ & 19.1 & $\mathrm{a}$ & $\mathrm{Y}$ \\
\hline Halosulfuron & 70 & $3-4$ tri & 18.1 & $\mathrm{a}$ & $\mathrm{Z}$ & 19.2 & $\mathrm{a}$ & $\mathrm{Z}$ & 17.0 & bcd & $\mathrm{Z}$ & 19.2 & $\mathrm{a}$ & $\mathrm{Z}$ \\
\hline Halosulfuron & 35 & $5-6$ tri & 17.7 & $\mathrm{a}$ & $\mathrm{Z}$ & 18.9 & $\mathrm{a}$ & $\mathrm{Z}$ & 16.6 & bc & $\mathrm{Z}$ & 19.7 & $\mathrm{a}$ & $\mathrm{Z}$ \\
\hline Halosulfuron & 70 & $5-6$ tri & 18.3 & $\mathrm{a}$ & $\mathrm{Z}$ & 18.9 & $\mathrm{a}$ & $\mathrm{Z}$ & 18.1 & cde & $\mathrm{Z}$ & 19.2 & $\mathrm{a}$ & $\mathrm{Z}$ \\
\hline Halosulfuron & 35 & $1^{\text {st }}$ flower & 18.0 & $\mathrm{a}$ & $\mathrm{Z}$ & 19.0 & $\mathrm{a}$ & $\mathrm{Z}$ & 19.5 & ef & $\mathrm{Z}$ & 19.2 & $\mathrm{a}$ & $\mathrm{Z}$ \\
\hline Halosulfuron & 70 & $1^{\text {st }}$ flower & 18.2 & $\mathrm{a}$ & $\mathrm{Z}$ & 19.3 & $\mathrm{a}$ & $\mathrm{YZ}$ & 21.3 & fg & $\mathrm{Y}$ & 19.4 & $\mathrm{a}$ & $\mathrm{YZ}$ \\
\hline Halosulfuron & 35 & $1^{\text {st }}$ pod & 18.5 & $\mathrm{a}$ & $\mathrm{Z}$ & 19.3 & $\mathrm{a}$ & $\mathrm{Z}$ & 20.7 & $\mathrm{f}$ & $\mathrm{Z}$ & 20.0 & $\mathrm{a}$ & $\mathrm{Z}$ \\
\hline Halosulfuron & 70 & $1^{\text {st }}$ pod & 18.2 & $\mathrm{a}$ & $\mathrm{Z}$ & 19.2 & a & $\mathrm{Z}$ & 22.7 & g & $\mathrm{Y}$ & 19.5 & $\mathrm{a}$ & $\mathrm{Z}$ \\
\hline Yield & & & & & & & & $\%$ & & & & & & \\
\hline Untreated check & & & 100 & $\mathrm{a}$ & & 100 & $\mathrm{~b}$ & & 100 & $\mathrm{a}$ & & 100 & $\mathrm{~b}$ & \\
\hline Halosulfuron & 35 & $1-2$ tri & 98 & $\mathrm{a}$ & $\mathrm{Y}$ & 112 & $\mathrm{a}$ & $\mathrm{Z}$ & 66 & bc & $\mathrm{X}$ & 109 & $\mathrm{ab}$ & $\mathrm{YZ}$ \\
\hline Halosulfuron & 70 & $1-2$ tri & 96 & $\mathrm{a}$ & $\mathrm{Y}$ & 112 & $\mathrm{a}$ & $\mathrm{Z}$ & 53 & de & $\mathrm{X}$ & 112 & $\mathrm{a}$ & $\mathrm{Z}$ \\
\hline Halosulfuron & 35 & $3-4$ tri & 99 & $\mathrm{a}$ & $\mathrm{Z}$ & 114 & $\mathrm{a}$ & $\mathrm{Z}$ & 69 & $\mathrm{~b}$ & $\mathrm{Y}$ & 108 & $\mathrm{ab}$ & $\mathrm{Z}$ \\
\hline Halosulfuron & 70 & $3-4$ tri & 98 & $\mathrm{a}$ & $\mathrm{Z}$ & 113 & $\mathrm{a}$ & $\mathrm{Z}$ & 54 & de & $\mathrm{Y}$ & 112 & $\mathrm{a}$ & $\mathrm{Z}$ \\
\hline Halosulfuron & 35 & $5-6$ tri & 97 & $\mathrm{a}$ & $\mathrm{Y}$ & 115 & $\mathrm{a}$ & $\mathrm{Z}$ & 63 & bcd & $\mathrm{X}$ & 110 & $\mathrm{ab}$ & $\mathrm{YZ}$ \\
\hline Halosulfuron & 70 & $5-6$ tri & 95 & $\mathrm{a}$ & $\mathrm{Y}$ & 111 & $\mathrm{a}$ & $\mathrm{Z}$ & 55 & de & $\mathrm{X}$ & 113 & $\mathrm{a}$ & $\mathrm{Z}$ \\
\hline Halosulfuron & 35 & $1^{\text {st }}$ flower & 100 & $\mathrm{a}$ & $\mathrm{Z}$ & 110 & $\mathrm{ab}$ & $\mathrm{Z}$ & 59 & bcd & $\mathrm{Y}$ & 106 & $\mathrm{ab}$ & $\mathrm{Z}$ \\
\hline Halosulfuron & 70 & $1^{\text {st }}$ flower & 99 & $\mathrm{a}$ & $\mathrm{Y}$ & 105 & $\mathrm{ab}$ & $\mathrm{YZ}$ & 44 & e & $\mathrm{X}$ & 116 & $\mathrm{a}$ & $\mathrm{Z}$ \\
\hline Halosulfuron & 35 & $1^{\text {st }}$ pod & 95 & a & $\mathrm{Z}$ & 106 & $\mathrm{ab}$ & $\mathrm{Z}$ & 57 & $\mathrm{~cd}$ & $\mathrm{Y}$ & 107 & $\mathrm{ab}$ & $\mathrm{Z}$ \\
\hline Halosulfuron & 70 & $1^{\text {st }}$ pod & 94 & $\mathrm{a}$ & $\mathrm{Z}$ & 108 & $\mathrm{ab}$ & $\mathrm{Z}$ & 45 & e & $\mathrm{Y}$ & 106 & $\mathrm{ab}$ & $\mathrm{Z}$ \\
\hline
\end{tabular}

${ }^{\mathrm{a}}$ Abbreviations: tri, trifoliate. ${ }^{\mathrm{b}}$ Non-ionic surfactant included at $0.25 \%$ and $0.5 \% \mathrm{v} / \mathrm{v}$ with the 35 and $70 \mathrm{~g} \cdot \mathrm{ai}^{\mathrm{h}} \mathrm{ha}^{-1}$ of halosulfuron, respectively. 
much as $4.4 \%, 3.0 \%, 4.1 \%, 7.3 \%$ and $8.7 \%$ at $1-2$ trifoliate, $3-4$ trifoliate, 5 - 6 trifoliate, $1^{\text {st }}$ flower and $1^{\text {st }}$ pod application timings, respectively (Table 4). In other studies halosulfuron applied POST at 2 - 3 trifoliate increased seed moisture content by $1.8 \%$ - 3\% in adzuki, cranberry and kidney bean but had no effect on the seed moisture content of black, otebo, pinto, small red Mexican and white beans [9].

\subsection{Seed Yield}

Halosulfuron applied POST at 35 and $70 \mathrm{~g} \cdot \mathrm{ai} \cdot \mathrm{ha}^{-1}$ had no adverse effect on seed yield of black, white and kidney bean at all application timings (Table 3). However, it decreased adzuki bean seed yield as much as $47 \%$, $46 \%, 45 \%, 56 \%$ and $55 \%$ at $1-2$ trifoliate, $3-4$ trifoliate, $5-6$ trifoliate, $1^{\text {st }}$ flower and $1^{\text {st }}$ pod application timings, respectively (Table 4). In other studies halosulfuron applied POST at 2 - 3 trifoliate reduced seed yield of adzuki bean as much as $68 \%$ and white bean as much as $9 \%$ but had no adverse effect on seed yield of black, cranberry, kidney, otebo, pinto and small red Mexican beans [9]. Sulfonylurea herbicides such as thifensulfuron applied POST caused as much as $89 \%$ yield reduction in yield and chlorimuron applied POST decreased seed yield as much as $93 \%$ in dry bean [14].

\section{Conclusion}

Halosulfuron applied POST at the proposed manufacturer's rate of $35 \mathrm{~g} \cdot \mathrm{ai} \cdot \mathrm{ha}^{-1}$ or twice that rate caused significant injury 1WAA in black, kidney and white beans. Generally, the injury decreased as the application timing was delayed from 1 - 2 trifoliate to $1^{\text {st }}$ pod stage. Crop injury was transient with minimal injury 4 WAA and no adverse effect on the shoot dry weight, plant height, seed moisture content and yield of black, white and kidney bean. Injury was significantly higher in adzuki bean compared to black, white and kidney bean at all application timings. Halosulfuron applied POST to adzuki bean at 1 - 2 trifoliate, 3 - 4 trifoliate, 5 - 6 trifoliate, $1^{\text {st }}$ flower and $1^{\text {st }}$ pod application timings caused severe injury, plant height reduction, shoot dry weight reduction, seed moisture content elevation, and seed yield reduction. Based on these results, there is potential for halosulfuron applied POST at $35 \mathrm{~g} \cdot \mathrm{ai}^{\cdot \mathrm{ha}^{-1}}$ after 3 - 4 trifoliate stage in black, white and kidney beans. However, there is not an adequate margin of crop safety for halosulfuron applied POST in adzuki bean at any of the application timings evaluated.

\section{Acknowledgements}

The authors would like to acknowledge Todd Cowan for his expertise and technical assistance in these studies. Funding for this project was provided in part by Gowan, Ontario Bean Growers, and the Agricultural Adaptation Council.

\section{References}

[1] Arnold, N.R., Murray, W.M., Gregory, J.E. and Smeal, D. (1993) Weed Control in Pinto Beans (Phaseolus vulgaris) with Imazethapyr Combinations. Weed Technology, 7, 361-364.

[2] Malik, V.S., Swanton, C.J. and Michaels, T.E. (1993) Interaction of White Bean (Phaseolus vulgaris) Cultivars, Row Spacing, and Seeding Density with Annual Weeds. Weed Science, 41, 62-68.

[3] Chikoye, D., Weise, S.F. and Swanton, C.J. (1995) Influence of Common Ragweed (Ambrosia artemisiifolia) Time of Emergence and Density on White Bean (Phaseolus vulgaris). Weed Science, 43, 375-380.

[4] Burnside, O.C., Ahrens, W.H., Holder, B.J., Wiens, M.J., Johnson, M.M. and Ristau, E.A. (1994) Efficacy and Economics of Various Mechanical plus Chemical Weed Control Systems in Dry Bean (Phaseolus vulgaris). Weed Technology, 8, 238-244.

[5] Bauer, T.A., Renner, K.A., Penner, D. and Kelly, J.D. (1995) Pinto Bean (Phaseolus vulgaris) Varietal Tolerance to Imazethapyr. Weed Science, 43, 417-424.

[6] Urwin, C.P., Wilson, R.G. and Mortensen, D.A. (1996) Responses of Dry Edible Bean (Phaseolus vulgaris) Cultivars to Four Herbicides. Weed Technology, 10, 512-518.

[7] Ontario Ministry of Agriculture, Food and Rural Affairs (2010) Guide to Weed Control. Publication 75, Toronto.

[8] Senseman, S.A. (2007) Herbicide Handbook. 9th Edition, Weed Science Society of America, Champaign, 458 p.

[9] Soltani, N., Shropshire, C. and Sikkema, P.H. (2012) Response of Dry Bean to Halosulfuron Applied Postemergence. Canadian Journal of Plant Science, 92, 723-728. http://dx.doi.org/10.4141/cjps2011-220 
[10] Stewart, C.L., Nurse, R.E., Gillard, C. and Sikkema, P.H. (2010) Tolerance of Adzuki Bean to Preplant-Incorporated, Pre-Emergence, and Post-Emergence Herbicides in Ontario, Canada. Weed Biology and Management, 10, 40-47. http://dx.doi.org/10.1111/j.1445-6664.2010.00365.x

[11] Powell, G.E. and Sprague, C.L. (2006) Tolerance of Six Classes of Dry Edible Bean and Adzuki Bean to PRE and POST Applications of Halosulfuron. North Central Weed Science Society, Milwaukee.

[12] Wall, D.A. (1995) Bentazon Tank-Mixtures for Improved Redwood Pigweed (Amaranthus retroflexus) and Common Lambsquarters (Chenopodium album) Control in Navy Beans (Phaseolus vulgaris). Weed Technology, 9, 610-616.

[13] Silvey, B.D., Mitchem, W.E., Macrae, A.W. and Monks, D.W. (2006) Snap Bean (Phaseolus vulgaris) Tolerance to Halosulfuron PRE, POST, or PRE Followed by POST. Weed Technology, 20, 873-876. http://dx.doi.org/10.1614/WT-05-046.1

[14] Sikkema, P.H., Soltani, N., Shropshire, C. and Cowan, T. (2004) Tolerance of White beans To Postemergence Broadleaf Herbicides. Weed Technology, 18, 893-901. http://dx.doi.org/10.1614/WT-03-043R3 
Scientific Research Publishing (SCIRP) is one of the largest Open Access journal publishers. It is currently publishing more than 200 open access, online, peer-reviewed journals covering a wide range of academic disciplines. SCIRP serves the worldwide academic communities and contributes to the progress and application of science with its publication.

Other selected journals from SCIRP are listed as below. Submit your manuscript to us via either submit@scirp.org or Online Submission Portal.
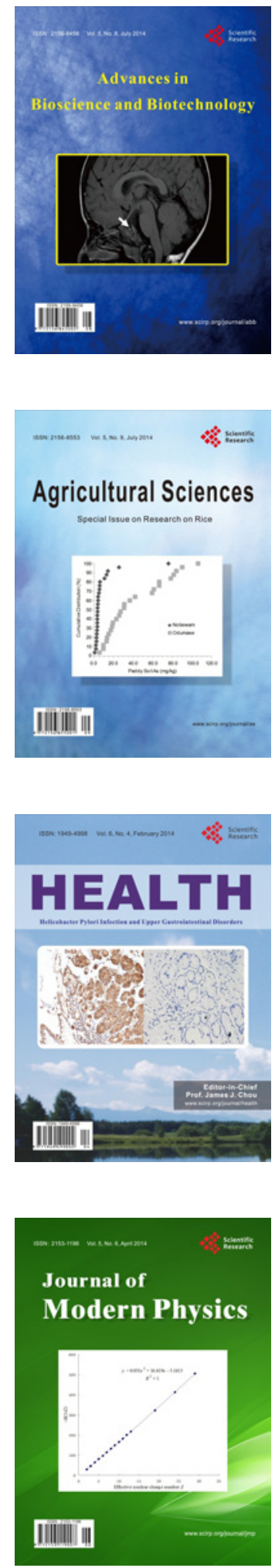
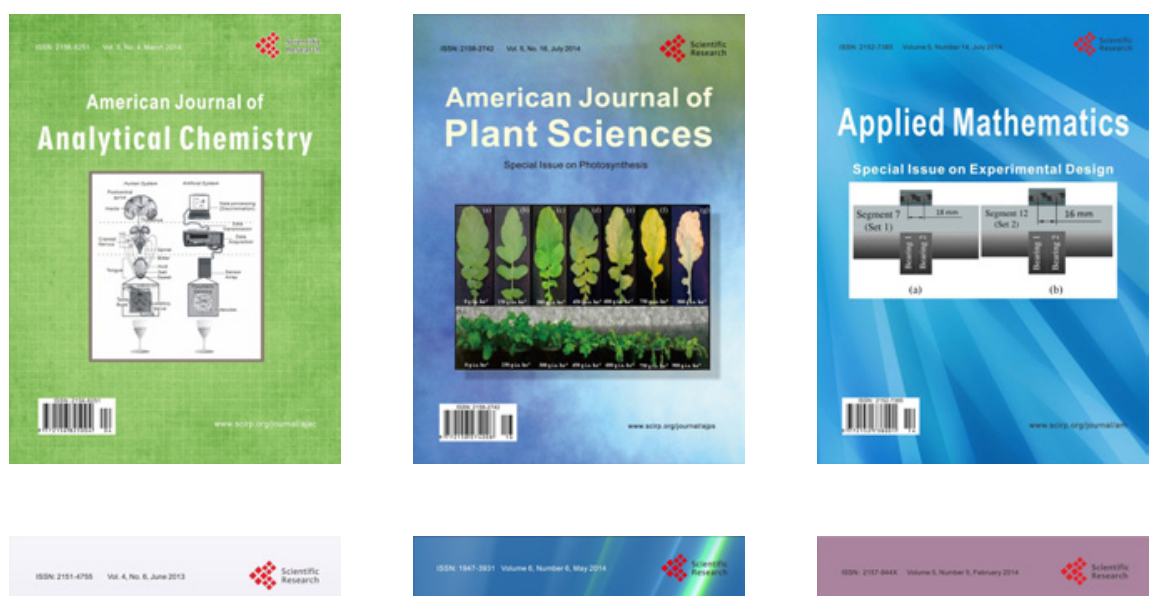

Creative Education
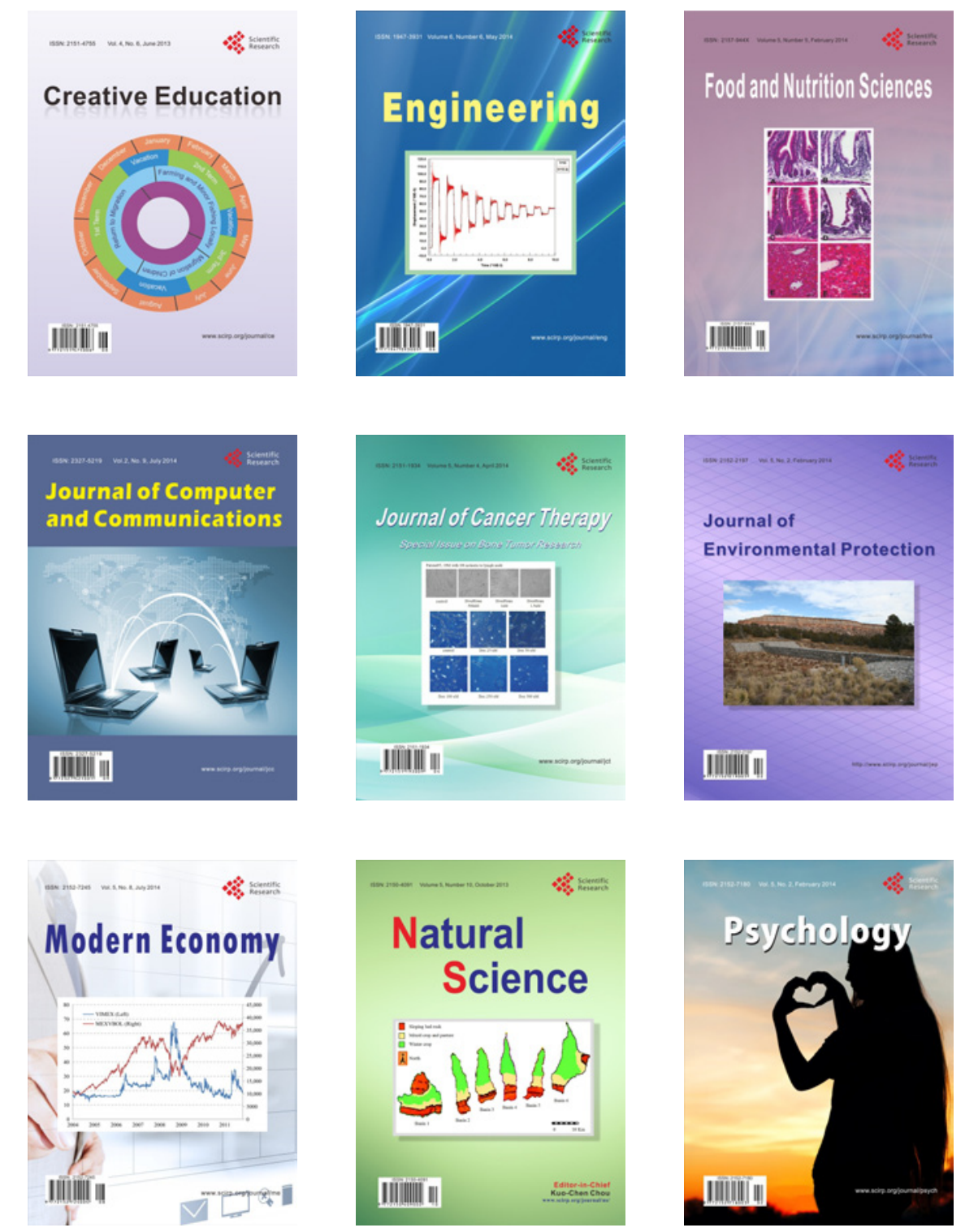